\title{
Neuronal Birth Order Identifies a Dimorphic Sensorineural Map
}

\author{
Jesús Pujol-Martí, ${ }^{1}$ Andrea Zecca, ${ }^{1 \star}$ Jean-Pierre Baudoin, ${ }^{1 \star}$ Adèle Faucherre, ${ }^{1}$ Kazuhide Asakawa, ${ }^{2}$ Koichi Kawakami, ${ }^{2}$ \\ and Hernán López-Schier ${ }^{1}$ \\ ${ }^{1}$ Laboratory of Sensory Cell Biology and Organogenesis, Centre de Regulació Genòmica, 08003 Barcelona, Spain, and ${ }^{2}$ Division of Molecular and \\ Developmental Biology, National Institute of Genetics, The Graduate University for Advanced Studies (SOKENDAI), Shizuoka 411-8540, Japan
}

Spatially distributed sensory information is topographically mapped in the brain by point-to-point correspondence of connections between peripheral receptors and central target neurons. In fishes, for example, the axonal projections from the mechanosensory lateral line organize a somatotopic neural map. The lateral line provides hydrodynamic information for intricate behaviors such as navigation and prey detection. It also mediates fast startle reactions triggered by the Mauthner cell. However, it is not known how the lateralis neural map is built to subserve these contrasting behaviors. Here we reveal that birth order diversifies lateralis afferent neurons in the zebrafish. We demonstrate that early- and late-born lateralis afferents diverge along the main axes of the hindbrain to synapse with hundreds of second-order targets. However, early-born afferents projecting from primary neuromasts also assemble a separate map by converging on the lateral dendrite of the Mauthner cell, whereas projections from secondary neuromasts never make physical contact with the Mauthner cell. We also show that neuronal diversity and map topology occur normally in animals permanently deprived of mechanosensory activity. We conclude that neuronal birth order correlates with the assembly of neural submaps, whose combination is likely to govern appropriate behavioral reactions to the sensory context.

\section{Introduction}

In aquatic environments, mechanical stimuli can convey information about the location and trajectory of obstacles, conspecifics, prey, and predators, allowing animals to optimize navigation or to escape from a threat (Dijkgraaf, 1963; Bleckmann and Zelick, 2009). The spatial distribution of sensory information is topographically mapped in the CNS by stereotypical connections between peripheral receptors and target neurons, which is essential for the accurate transmission of environmental stimuli to processing centers in the brain (Luo and Flanagan, 2007). For example, hydromechanic variations along the body of fishes and amphibians are captured by external mechanosensory organs called neuromasts, which collectively form the lateral line (Ghysen and Dambly-Chaudière, 2007). Locally acquired mechanical

\footnotetext{
Received 0ct. 12, 2011; revised Jan. 5, 2012; accepted Jan. 6, 2012.

Author contributions: J.P.-M. and H.L.-S. designed research; J.P.-M., A.Z., J.-P.B., and A.F. performed research; K.A. and K.K. contributed unpublished reagents/analytic tools; J.P.-M., J.-P.B., A.F., and H.L.-S. analyzed data; J.P.-M. and H.L.-S. wrote the paper.

*A.Z. and J.-P.B. are equally contributing authors.

This research was supported by funds provided by the Agència de Gestió d'Ajuts Universitaris i de Recerca (AGAUR) (2009-SGR-305) and by the European Research Council (ERC-2007-StG SENSORINEURAL). J-P.B. was supported by the Fondation pour la Recherche Médicale of France, A.F. by an Intra-European Marie Curie Fellowship, and A.Z. by a Fellowship of the AGAUR. J.P-M. is a Formación de Personal Investigador fellow and H.L-S. a Ramón y Cajal Investigator of the Ministerio de Ciencia e Innovación of Spain. K.A. and K.K. were funded by the National BioResource Project and by grants from the Ministry of Education, Culture, Sports, Science and Technology of Japan. We thank J. C. Liao for communicating results before publication. C. Moens for mutant fish, and S. J. Smith and R. Tsien for DNA constructs. We thank The Advanced Light Microscopy Unit of the Centre de Regulació Genòmica for advice.

Correspondence should be addressed to Hernán López-Schier, Laboratory of Sensory Cell Biology and Organogenesis, Centre de Regulació Genòmica, Dr. Aiguader 88, 08003 Barcelona, Spain. E-mail: hernan.lopez-schier@helmholtz-muenchen.de.

DOI:10.1523/JNEUROSCI.5157-11.2012

Copyright $\odot 2012$ the authors $\quad 0270-6474 / 12 / 322976-12 \$ 15.00 / 0$
}

signals by the sensory elements of the neuromast, called hair cells, are transmitted to bipolar afferent neurons that project central axons to the medial octavolateralis nucleus of the hindbrain (Claas and Münz, 1981; Alexandre and Ghysen, 1999; Ghysen and Dambly-Chaudière, 2007). This first mechanosensory relay contains a somatotopic neural map, in which the afferent central projections are stratified along a dorsomedial-ventrolateral axis that reflects the spatial distribution of the neuromasts (Claas and Münz, 1981; Alexandre and Ghysen, 1999; Ghysen and DamblyChaudière, 2007). Lateral-line somatotopy suggests that this sensory system builds a continuous neural map (Alexandre and Ghysen, 1999; Luo and Flanagan, 2007).

The lateral line possesses a dual function. It plays a major role in navigation, schooling, rheotaxia, prey detection, and hunting (Montgomery et al., 1997, 2000; Coombs and Patton, 2009). This set of behaviors necessitates continuous input and involves fine and complex processing of the hydrodynamic field. The lateral line also mediates very fast responses to sudden mechanical stimuli, such as the C-start escape behavior that is triggered by the activation of a reticulospinal command neuron called Mauthner cell (McHenry et al., 2009). Because the value of the escape response is its near immediate onset after potentially threatening stimuli, it is best served by avoiding high-order processing. It is currently not understood how the lateralis neural circuit is built to subserve these contrasting behaviors. Here we investigate this issue by characterizing the assembly of the posterior lateralis sensorineural map.

\section{Materials and Methods}

Zebrafish strains and husbandry. Zebrafish were maintained under standardized conditions and experiments were conducted in embryos of un- 
determined sex in accordance with protocols approved by the Parc de Recerca Biomèdica de Barcelona's Ethical Committee of Animal Exper-

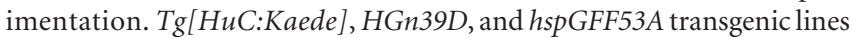
have been published previously (Sato et al., 2006; Faucherre et al., 2009; Pujol-Martí et al., 2010). The hspGFFDMC130A was generated by random integration of an enhancer-trap construct (Asakawa et al., 2008). The atoh1a_fh282 mutant strain carries a missense mutation, aa126 arginine to tryptophan, and was obtained from C. Moens (Fred Hutchinson Cancer Research Center, Seattle, WA). The tmie_ru01 mutant fish were described previously (Gleason et al., 2009; Faucherre et al., 2010).

Selection of mutants. Wild-type animals and homozygous mutants for tmie and atohla were sorted based on DiASP incorporation into hair cells of the lateral line. Homozygous mutants for tmie were genotyped by amplification using the following primers and sequencing: For (forward): $5^{\prime}$-CCAG CAGCTCTCGTAACCTC-3'; Rev (reverse): 5'-CCGCCATCACCAGT CTATTT-3'.

Plasmid DNA constructs and injections. For cloning the SILL enhancer, the $2 \mathrm{~kb}$ upstream of the HGn39D insertion was amplified from genomic DNA and cloned in the Tol2kit 219 plasmid using the following primers: EnUp-attb4-For: 5' -GGGG ACA ACT TTG TAT AGA AAA GTT G TAAAGAAATGTCAAGTGTTT-3'; EnUp-attB1-Rev: 5'-GGGG AC TGC TTT TTT GTA CAA ACT TG GTTGGGATGGTGTACAGTAT-3'.

The $2 \mathrm{~kb}$ downstream of the HGn39D insertion was amplified and cloned in the Tol2kit 220 plasmid using the following primers: EnDoattB2-For: 5'-GGGG ACA GCT TTC TTG TAC AAA GTG G CC ATCCCAACTCACTCACTATT-3'; EnDO-attB3-Rev: 5'-GGGG AC AAC TTT GTA TAA TAA AGT TG C CTGACATTTTCCGGAACAGG-3'.

The hsp70:mCherry-SILL (SILL1), hsp70:Gal4VP16-SILL (SILL2) and hsp70:MCS-SILL constructs were obtained using the Tol2 kit (Kwan et al., 2007). Entry vectors were generated as described in the Invitrogen Multisite Gateway manual. PCRs were performed using primers to add att sites onto the end of DNA fragments, using Platinum Pfx (Invitrogen). The pEntry vectors containing the hsp70 promoter, the mCherry, the Gal4VP16, and the MCS (multiple-cloning site) are from the Tol 2 kit. To generate the construct, VAMP-GFP was amplified using the UAS:VAMP-GFP;UAS:Kir2.1 plasmid as a template (Hua et al., 2005), with the following primers: For: 5'-AGAC GATATCATGTCTGCCCCAGATGCT-3';

5'-TATGGATATCTTACTTGTACAGCTCGTC-3'.

The PCR product was digested by EcoRV and cloned into the hsp70: MCS-SILL construct.

Generation of transgenic zebrafish. To generate the Tg[SILL1] stable transgenic line, $20 \mathrm{pg}$ of the Tol2-expression clone and $20 \mathrm{pg}$ of the transposase synthetic RNA were simultaneously injected into one-cell stage wild-type eggs. The resulting embryos were raised to adulthood and incrossed for visually screening of germline transmission of the transgene. For mosaic expression in neurons, $25-30 \mathrm{pg}$ of DNA was injected into embryos at the one- or two-cell stage.

Whole-mount in situ hybridization. We generated labeled RNA probes by in vitro transcription using the DIG/Fluor RNA labeling Mix (Roche). Embryos were fixed in $4 \%$ paraformaldehyde in PBS overnight at $4{ }^{\circ} \mathrm{C}$ and whole-mount in situ hybridizations were carried using standard protocols.

To characterize cntnap 2 expression, the following antisense probe was used: Ensemble Zv9 ENSDART00000081960, nucleotides 3049-3357.

Labeling and imaging. Neuronal labeling by fluorescent dextrans was performed as previously described (Pujol-Martí et al., 2010). We used a redfluorescent dextran [tetramethylrhodamine, $3000 \mathrm{MW}$ (molecular weight), anionic, fixable, Invitrogen ref.D3308) and a magenta-fluorescent dextran (Alexa Fluor 647, 10,000 MW, anionic, fixable, Invitrogen ref.D22914). Imaging was done $1 \mathrm{~d}$ after dextran injection. In all the samples, we imaged the dextran injection site to confirm that only neurons innervating an individual neuromast were labeled. We analyzed samples in which the neuronal labeling was specific to the selected neuromast, with the exception of the dextran injections into the D2 neuromast, in which neurons innervating the more posterior and dorsal neuromasts were often also labeled.

Imaging, birthdating and anatomical analysis. For BAIT (Birthdating combined with Anatomical analysis by Incorporation of Tracers) experiment, $\mathrm{Tg}$ [HuC:Kaede] eggs were kept in the dark. At the $28 \mathrm{~h}$ postfertilization (hpf) stage, embryos were exposed to $405 \mathrm{~nm}$ light for $2 \mathrm{~min}$ and subsequently kept in the dark for $3 \mathrm{~d}$. Neurons innervating terminal neuromasts were labeled by magenta-fluorescent dextran and the posterior lateralis ganglion was imaged $1 \mathrm{~d}$ later. For imaging, live samples were anesthetized and mounted onto a glass-bottom small Petri dish (MatTek) and covered with 1\% low-melting-point agarose with diluted anesthetic. Images were acquired with a Leica TCS SP5 inverted confocal laser scanning microscope with a $20 \times$ air objective, or a $40 \times$ oilimmersion objective. $Z$-stacks of central and peripheral axons consisted of $1 \mu \mathrm{m}$ spaced images. $Z$-stacks of neuronal somata consisted of $2.5 \mu \mathrm{m}$ spaced images. Three-dimensional reconstructions and cropping of $z$-stacks were done with Imaris software (Bitplane).

$Q$ uantification of soma volume and peripheral axon diameter. To measure soma volume, a surface reconstruction of each soma imaged was done using Imaris, with a surface area detail level of $0.5 \mu \mathrm{m}$ and a thresholding based on absolute intensity. Threshold was manually adjusted to optimally match the surface reconstruction with the fluorescent signal. Imaris automatically quantifies the volume of the surface reconstruction. To measure peripheral axon diameter, we imaged a $\sim 100 \mu \mathrm{m}$ segment of the peripheral axon just anterior to the peripheral arborization at the level of the neuromast using a Leica TCS SP5 inverted confocal microscope with a $20 \times$ air objective, with Zoom: 8 . Diameters were measured at ten equidistant locations of the imaged axonal segment and the average was calculated.

Laser-mediated cell ablation. For neuronal ablation we used a Micropoint laser system (Photonic Instruments Inc.) mounted on an Olympus IX81 inverted microscope equipped with a $40 \times$ air objective. $H G n 39 D$ embryos at 28-30 hpf were anesthetized, mounted on a glass-bottom dish (MatTek), and covered with methylcellulose. A train of laser pulses was repeatedly applied to the posterior lateralis ganglion until all EGFP fluorescence disappeared. Embryos were allowed to recover for $2 \mathrm{~h}$ and then assessed for the presence of EGFP in the region of the posterior ganglion. Total ablation occurred in samples with no green-fluorescent signal in that region. One day later, we chose samples with new neurons forming a ganglion. We next labeled neurons projecting from the terminal neuromasts at 5-6 d postfertilization ( $\mathrm{dpf}$ ) by injecting fluorescent dextran at the neuromasts. We did the same in non-ablated controls.

\section{Results}

\section{HGn39D is an insertion in Cntnap2a}

We have previously reported that the zebrafish transgenic line $H G n 39 D$ expresses EGFP in all the afferent neurons of the lateral line (Faucherre et al., 2009). We sought to determine the integration site in $H G n 39 D$ by sequencing genomic DNA flanking the transgene. This revealed a single insertion on chromosome 24 within a locus coding for a zebrafish homolog of the contactinassociated protein-like 2/Caspr2 (which we herein call Cntnap2a to differentiate it from a second Cntnap2 (Cntnap2b) located on chromosome 2) (Fig. 1A,B). CNTNAPs are members of the Neurexin family of neuronal cell-adhesion proteins, which have been associated with autism and epilepsy in humans and in the mouse (Peñagarikano et al., 2011). Next, we used whole-mount in situ hybridization in $3 \mathrm{dpf}$ zebrafish embryos to determine the expression pattern of EGFP in HGn39D and of the endogenous Cntnap2a, which revealed strong expression of both genes in the anterior and posterior lateralis ganglia (Fig. 1C,D). Additional weak expression of Cntnap2a may be present throughout the CNS. This result suggests that the specific expression in HGn39D may be replicated with new transgenes. Toward this aim, we characterized regulatory regions of Cntnap2a in experimental constructs that were tested by transient transgenesis by injecting them as plasmids into fertilized zebrafish eggs. We started by cloning $2 \mathrm{~kb}$ fragments of genomic DNA flanking the insertion site in HGn39D. We tried several combinations of these genomic fragments placed upstream and/or downstream of the heatshock-70 promoter followed by a cytoplasmatic version of the monomeric red-fluorescent protein mCherry. DNA fragments 
A

CACCATAAGAAACTAATGGGTTTCCAGAC TATATTCTTCCATTAGACAGAAAACATCC AGATATACTGTACACCATCCTAACTCACT TACTATTTGTATTCACTGCAGTTATT TATATCTAATTCTACAAATTCATGTTAAA

C

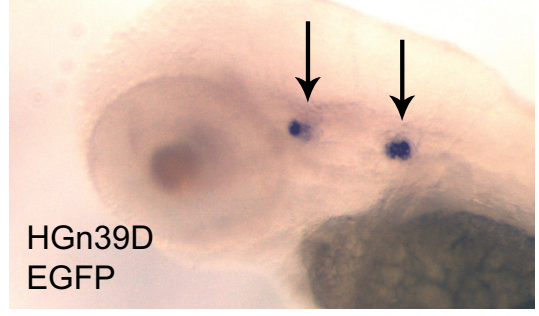

B

Contigs

BLAT/BLAST hits Ensemb//Havana

Zebrafish CDNA

Gene Legend

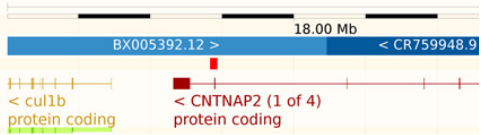

Reverse strand

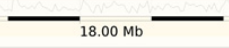

D

wt

cntnap2
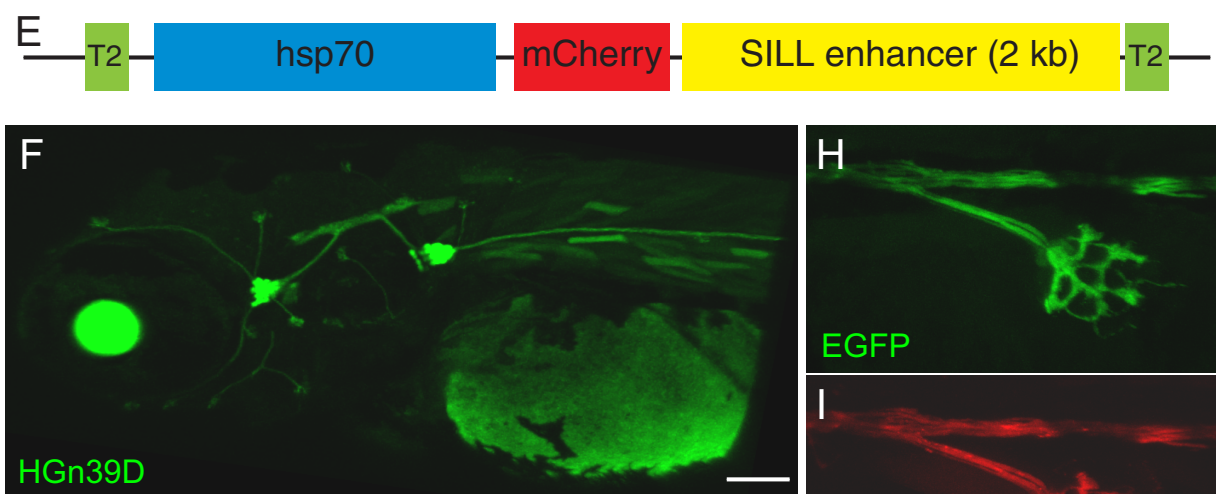

\section{G}

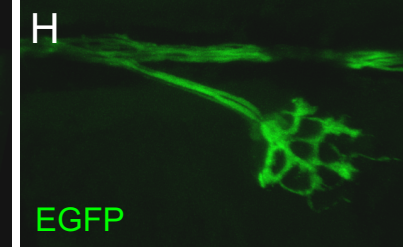

HGn39D
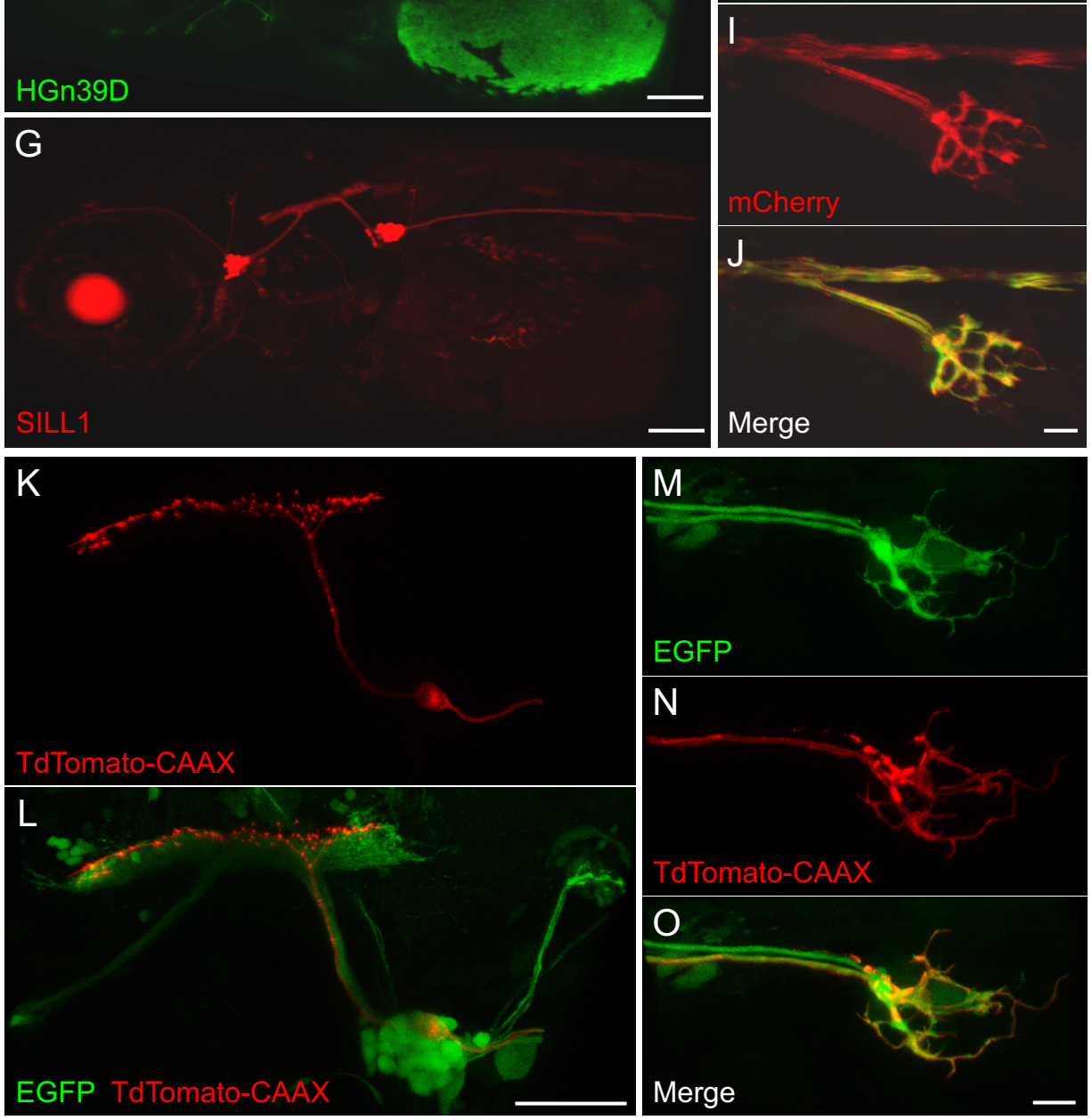

Figure 1. The SILL enhancer drives gene expression in the lateralis afferent neurons. $A$, The HGn39D enhancer-trap site indicated by red nucleotides. $B$, BLAST (basic local alignment search tool) result shows the integration within (ntnap2a. C, D, Whole-mount in situ hybridization of the EGFP transcripts in $3 \mathrm{dpf} H G n 39 D$ transgenic fish (C), and of the (ntnap2 (Figure legend continues.) 
upstream of the insertion site were not driving any visible transgene expression. However, a $2 \mathrm{~kb}$ downstream fragment placed downstream of mCherry drove robust gene expression in lateralis afferent neurons (data not shown). We named this construct SILL1 (for Sensory Innervation of the Lateral Line number 1) (Fig. $1 E$ ). We subsequently generated a stable transgenic line bearing the SILL1 construct, which fully recapitulated the expression pattern of the original enhancer-trap line (Fig. $1 F-J$ ). We also constructed SILL2 by replacing mCherry with a Gal4-VP16 transcription factor. Injecting the SILL2 plasmid into eggs from the Tg[UAS:TdTomato-CAAX; HGn39D] stable transgenic line activated mosaic expression of the red-fluorescent protein in the afferent neurons of the lateral line (Fig. $1 \mathrm{~K}-\mathrm{O}$ ).

Lateralis afferent neurons are structurally diverse and diverge in the hindbrain

Stochastic expression of SILL1 by DNA injection allowed us to obtain unprecedented resolution of individual lateralis afferent neurons (Fig. 2A). We selected for analysis animals in which single mCherry-labeled axons were resolvable in the hindbrain. We combined this analysis with neuronal labeling with fluorescent dextrans (see below). High-resolution in vivo threedimensional imaging and quantifications revealed that the central projection of each neuron branches into $\sim 60$ bulged spines or boutons (Fig. $2 A-D$ ), which likely represent synaptic contacts with second-order targets because they enriched a well characterized presynaptic marker (Fig. 2E-G) (Hua et al., 2005). In the course of this systematic anatomical study, we noticed that some central axons consistently presented a ventral-pointing indentation in their rostral ramus (Fig. $2 A$ ). To know whether this indentation identified a particular neuronal population, we labeled all the neurons from a neuromast by injecting dextrans in the vicinity of their peripheral arborization below the hair cells. Dextran uptake and retrograde transport highlights the entire neuron, including fine details of their central projections (Fig. $2 \mathrm{H}$ ). Injections of magenta-dextran in terminal neuromasts resulted in the labeling of up to four neurons (Fig. 2I), whose identity as afferent was established by the localization of their soma within the posterior lateralis ganglion (Fig. $2 \mathrm{H}$ ). We consistently observed that only around half of the labeled axons presented an indentation. Indented axons always projected dorsally in the lateralis column of the hindbrain (Fig. 2I-L). Neurons without the indentation had ventrolateral projections that appeared thinner (Fig. 2I,L). Next, we asked whether the position of the indentation was conserved for all axons. For this purpose we used a triple transgenic line Tg[SILL1; hspGFFDMC130A; UAS: EGFP] that expressed EGFP under the control of the Gal4 in the Mauthner cell and mCherry in all the lateralis afferent neurons (Fig. 2M). The stereotyped localization and orientation of the Mauthner cell in the hindbrain was used as three-dimensional

$\leftarrow$

(Figure legend continued.) transcripts in a wild-type specimen (D). Arrows indicate the anterior and posterior lateralis ganglia. $\boldsymbol{E}$, Schematic representation of the SILL1 construct. $\boldsymbol{F}, \mathbf{G}$, Maximal projections of lateral views showing the expression pattern of EGFP in $H G n 39 D(F)$ and of mCherry in Tg[SILL1] (G) at $3 \mathrm{dpf}$. H-J, Maximal projections of the peripheral axon arbor of Tg[HGn39D; SILL1] double transgenicfish at $3 \mathrm{dpf}$ showing coexpression of EGFP $(\boldsymbol{H})$ and $\mathrm{mCherry}(\boldsymbol{(})$. Merge is depicted in $J$ J $K, L$, Maximal projections of the posterior lateralis ganglion and central axons showing TdTomatoCAAX ( $\boldsymbol{K}$ ) and EGPF ( $\boldsymbol{L}$ ) expression in a $5 \mathrm{dpf}$ Tg[HGn39D; UAS:TdTomato-CAAX] double transgenic fish injected with the SILL2 construct that contains a Gal4-VP16 transcriptional activator. $\mathbf{M - 0}$, Tg[HGn39D; UAS:TdTomato-CAAX] double transgenic fish injected with the SILL2 construct expressing EGFP (M) and TdTomato-CAAX ( $\boldsymbol{M}$ ). Merge is depicted in (0). Scale bars: $F, G, L, 100 \mu \mathrm{m} ; J, 0,10 \mu \mathrm{m}$. In all the panels and figures, dorsal is up and anterior is left. landmark (Eaton et al., 1977; Kimmel et al., 1981). Maximal projections of confocal stacks showed that the lateral dendrite of the Mauthner cell invades the center of the lateralis column (Fig. $2 M)$. Medial-to-lateral progression of consecutive focal planes at higher magnification showed that the Mauthner's lateral dendrite coincides with the indentation of the dorsal-projecting axons (Fig. $2 \mathrm{~N}-\mathrm{Q}$ ). The indentation and projection pattern along the dorsoventral axis in the hindbrain suggest the existence of lateralis neuronal subclasses in the zebrafish larva.

\section{Neuronal subclassification based on contacts with a central target}

Lateralis afferents input monosynaptically the Mauthner cell in the zebrafish and other species (Zottoli and Van Horne, 1983; Kimmel et al., 1990). To ask whether the Mauthner cell is a direct output neuron of all lateralis afferent neurons, we injected magenta-dextran in the L1 neuromast of Tg[SILL1; hspGFFDMC130; UAS:EGFP] triple transgenics. This experiment showed that only the dorsal-projecting axons with an indentation contact en passant the lateral dendrite of the Mauthner cell (Fig. $3 A-C$ ), whereas ventral-projecting neurons do not, further supporting our subclassification of neurons. In the trunk of zebrafish larva, the lateral line is formed by neuromasts that derive from at least four primordia. Two primordia give rise to the dorsal and two to the posterior neuromasts. Posterior and dorsal neuromasts are subdivided into those originating from first primordia (early-born/primary neuromasts: respectively L1-terminal and D1), and those that are formed by second primordia (late-born/ secondary neuromasts: LII.1-LII.4 and D2-D4). Primary neuromasts contain hair cells that are plane polarized parallel to the anteroposterior body axis of the fish, whereas hair cells in secondary neuromasts are orthogonally oriented. The protracted development and structural diversity of the lateral line motivated us to ask whether all the neuromasts send direct projections to the Mauthner cell. For this purpose, we systematically injected dextrans in neuromasts identified by position as early-born/primary or late-born/secondary in the posterior and dorsal branches of the lateral line. Next, we assessed their projections in the triple transgenic line Tg[SILL1; hpsGFFDMC130; UAS:EGFP] (Fig. 3D-G). This experiment showed that no neuron from representative secondary neuromasts project to the Mauthner cell, whereas, injected primary neuromasts contained neurons that contact the lateral dendrite of the Mauthner, and neurons that do not $(p<0.05$, two-tailed Fisher's exact test) (Fig. $3 J)$ (Sheskin, 2004). Identical results were obtained by stochastic labeling of neurons by DNA injection ( $p<0.05$, two-tailed Fisher's exact test) (Fig. $3 H-J$ ), suggesting that the projection differences were not due to an effect of dextran incorporation into the neurons. The evidence from these experiments shows an asymmetric monosynaptic input of the lateral line to the Mauthner cell, with a disproportionate contribution from early-born/primary neuromasts.

There is a temporal progression of lateralis neurogenesis in the zebrafish embryo (Pujol-Martí et al., 2010; Sarrazín et al., 2010; Sato et al., 2010). Therefore, one possibility to explain neuronal heterogeneity is that neurons with the indentation or direct contact with the Mauthner cell are born at different times than those without it. However, over time late-born neurons may mature to resemble first-born. To test this possibility, we followed samples in which both neuronal classes from a single neuromast were labeled, and saw no differences in their central projections or contacts with the Mauthner cell over a $7 \mathrm{~d}$ period (Fig. $3 \mathrm{~K}$ ). To rule out an effect of dextrans on the normal long-term behavior of the axons, we also labeled the neurons from terminal neuromasts in naive HGn39D transgenic juveniles at $20 \mathrm{dpf}$ and 

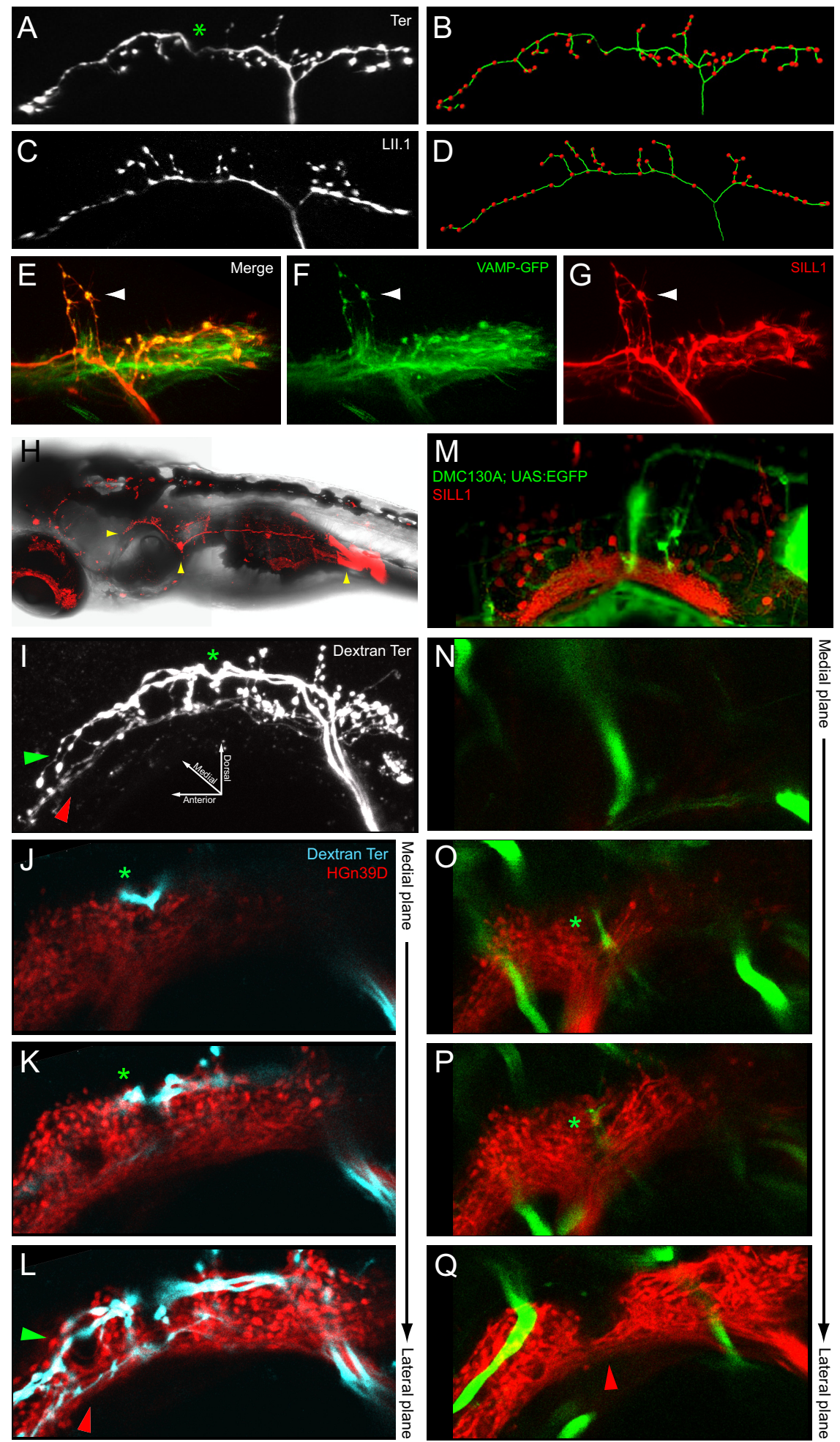

Figure 2. Structural diversity of lateralis afferent central projections. $\boldsymbol{A}-\boldsymbol{D}$, Central projection of an individual afferent neuron innervating a terminal neuromast (Ter) $(\boldsymbol{A})$ or a Lll.1 neuromast $(\boldsymbol{C})$ at $6 \mathrm{dpf}$. The neurons were labeled with SILL1 DNA in $\boldsymbol{A}$ and by red-dextran in $\boldsymbol{C}$. $\boldsymbol{B}$ and $\boldsymbol{D}$ are skeletonized drawings of the central projections in $\boldsymbol{A}$ and $\boldsymbol{C}$, respectively. The green filaments show the central axons main rami and their minor branches, the red dots represent the bulged spines. Asterisk indicates the indentation in the rostral ramus. $E-G$, Central projections of the afferent neurons colabeled with mCherry and the presynaptic marker VAMP-GFP (by coinjection of SILL1 and constructs) at $4 \mathrm{dpf}$ (Hua et al., 2005). VAMP-GFP is enriched in the bulged spines (arrowhead). $\boldsymbol{H}, \mathrm{A} 7$ dpf larva imaged with bright-field and fluorescent light, showing lateralis afferent neurons innervating LII. 1 labeled by reddextran. Arrowheads indicate the central projections, neuronal soma and injection site (from left to right sequence). $\boldsymbol{I}-\boldsymbol{L}$, (entral projections of lateralis afferent neurons from the terminal neuromasts, labeled by magenta-dextran at $6 \mathrm{dpf}$. $I$ is a maximal projection. $\boldsymbol{J}-\boldsymbol{L}$ are medial-to-lateral progression of consecutive confocal planes. The lateralis column is shown in red using HGn39D. The asterisk indicates the indentation. Green and red arrowheads indicate dorsal projections with indentation and observed them $1 \mathrm{~d}$ later. Again, we saw the presence of both neuronal subclasses (Fig. $3 L$ ).

\section{Biased axonal projection pattern of} large and small neurons

Having shown neuronal subclassification in relation to central projections and contact with the Mauthner cell, we next decided to search for additional differences between these neurons. Because dextran uptake eventually decorates the entire neuron, we looked at potential differences in the ganglion and peripheral axons. Within the ganglion we consistently found neurons with large and small somata (Fig. $4 A, B)$. We plotted the neurons in a twodimensional space in which each dot represented the soma volume of individual neurons. The data were then grouped by both the innervated neuromast and the labeling method. The distribution of neurons showed a clear distribution into two groups. We then arbitrarily set a mark of $1500 \mu \mathrm{m}^{3}$ because it is infrequently represented in the volume distribution, to classify neurons as large or small. This classification showed that large-soma neurons were always located dorsally in the ganglion, suggesting that they are older. A systematic anatomical characterization and quantification revealed that large-soma neurons projected exclusively from terminal neuromasts, whereas neurons from non-terminal neuromasts were homogeneously small (Fig. $4 C, D)$. We next assessed the relationship between the volume of the neuronal soma, the diameter of the axon, and contacts with the Mauthner cell in single neurons marked with the SILL1 construct. All large-soma neurons bore peripheral axons of larger diameter (Fig. $4 E-H$ ). We found that every large neurons made a direct contact with the Mauthner cell, whereas we could further subclassify small neurons into those that did and those that did not contact the Mauthner. This conclusion is based on a two-tailed Fisher's exact test, with a $p<0.05$ (Fig. $4 E-H$ ).

\section{Neuronal projections and birth date}

To ask about the timing of neuromast innervation relative to neuronal size, we la-

ventrolateral projections without indentation, respectively. $\mathbf{M}-\mathbf{Q}$, Spatial relationship between the Mauthner cell and the lateralis column in a Tg[SILL1; hspGFFDMC130A; UAS:EGFP] triple transgenic at $6 \mathrm{dpf}$. $\boldsymbol{M}$ is a maximal projection. $\mathbf{N}-\mathbf{Q}$ are medial-to-lateral progression of consecutive confocal planes. The asterisk shows the distal tip of the Mauthner cell's lateral dendrite. The red arrowhead indicates ventrolateral projections from lateralis neurons without the indentation. 

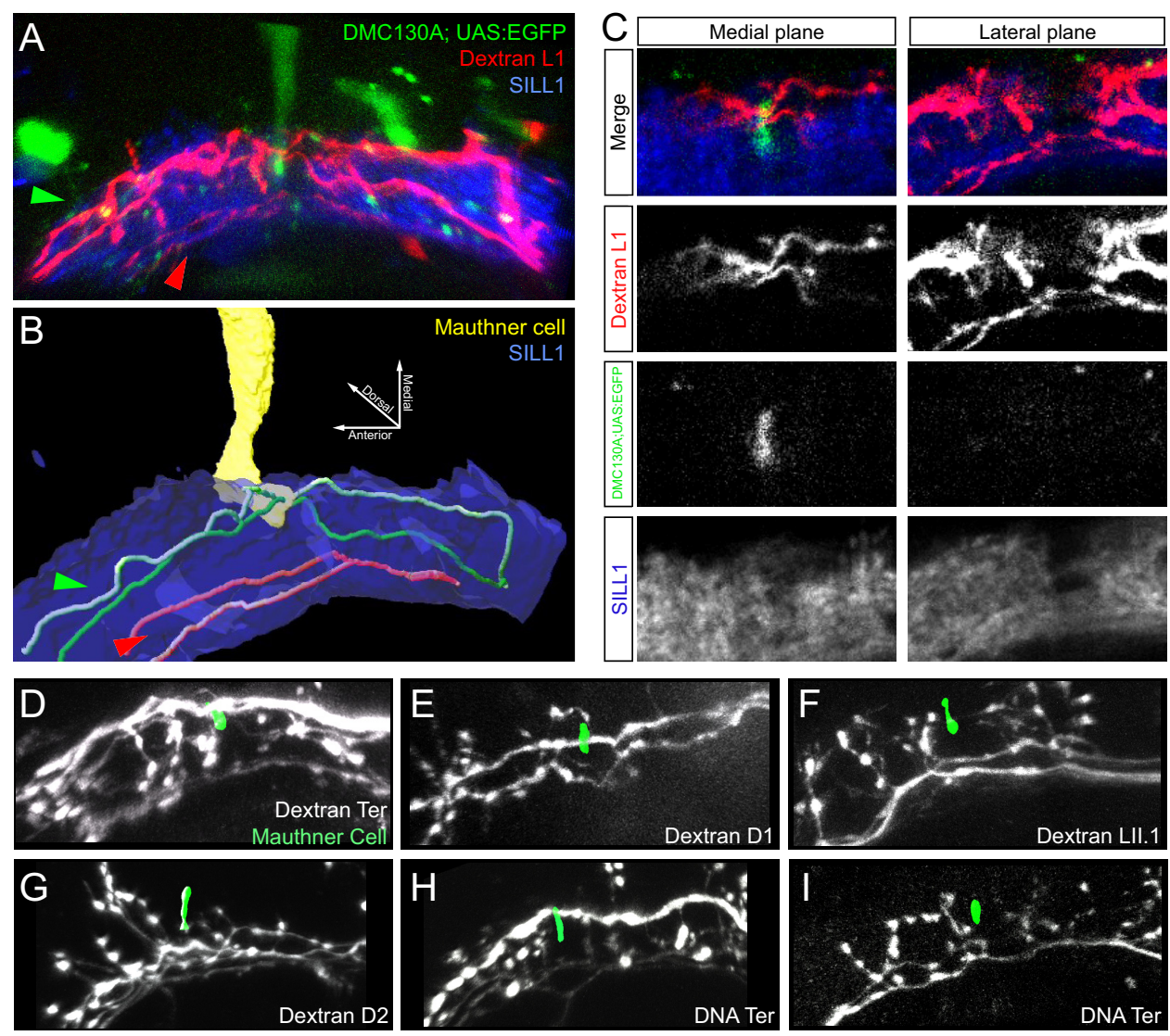

$\mathrm{J}$

\begin{tabular}{|cccccc|}
\hline $\begin{array}{c}\text { Innervated } \\
\text { neuromast }\end{array}$ & $\begin{array}{c}\text { Time of } \\
\text { development }\end{array}$ & $\begin{array}{c}\text { N neurons } \\
\text { Contacting M-cell } \\
\text { Dextran / DNA }\end{array}$ & $\begin{array}{c}\text { N neurons } \\
\text { Non-contacting M-cell } \\
\text { Dextran / D NA }\end{array}$ \\
L1 & Primary & 22 & 6 & 7 & 0 \\
Ter & Primary & 5 & 3 & 13 & 1 \\
LII.I & Secondary & 0 & 0 & 18 & 0 \\
LII.2 & Secondary & 0 & 0 & 0 & 1 \\
LII.3 & Secondary & 0 & 0 & 0 & 1 \\
D2 & Secondary & 0 & 0 & 13 & 0 \\
& & & & 9 & 3 \\
\hline
\end{tabular}
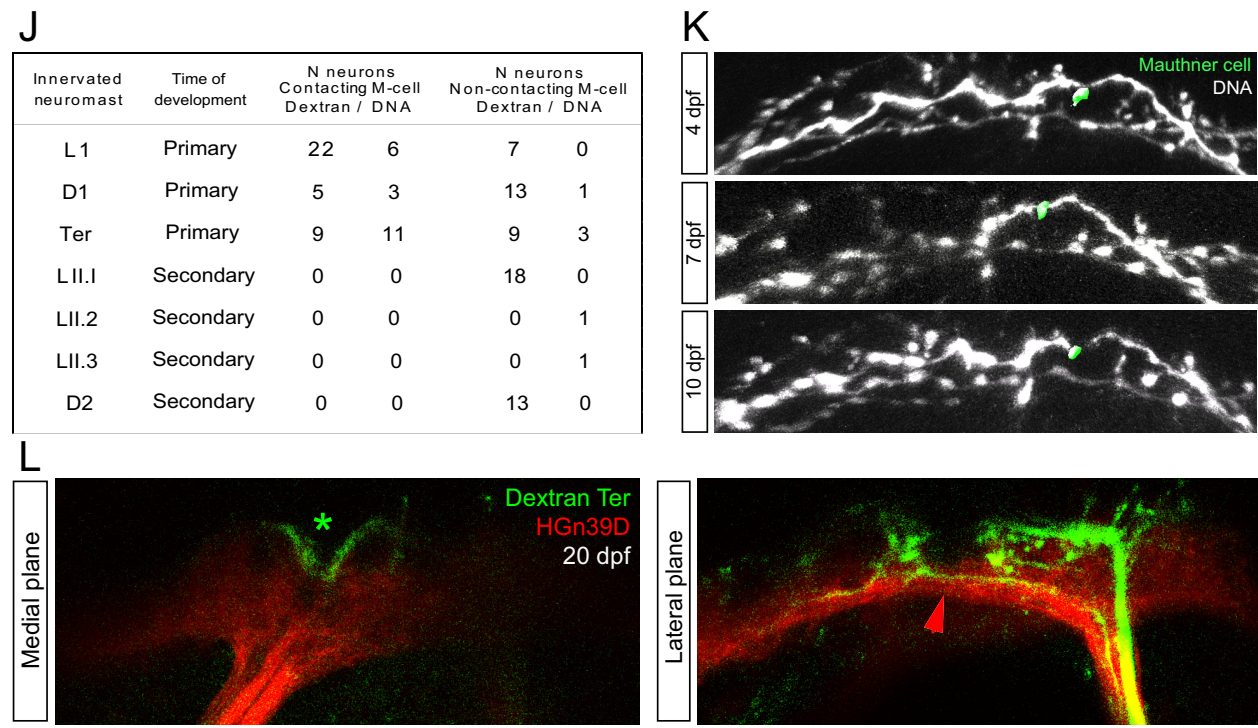

Figure 3. Neuronal subclassification based on contacts with the Mauthner cell. $A-C$, Central projections from lateralis afferent neurons of the L1 neuromast (early-born/primary neuromast) labeled by magenta-dextran at $6 \mathrm{dpf}$, in a Tg[SILL1; hspGFFDMC130A; UAS:EGFP] triple transgenic animal. $\boldsymbol{A}$ is a maximal projection; $\boldsymbol{B}$ is a snapshot of a three-dimensional reconstruction from the data shown in $A$. C, A detailed view of the central projections toward the Mauthner cell (M-cell) are shown in a medial-to-lateral progression of confocal planes. Green and red arrowheads indicate, respectively, dorsal projections contacting the Mauthner cell and ventrolateral projections non-contacting the Mauthner cell. $D-I$, Central axons from afferent neurons projecting from early-born/primary neuromasts (D1, terminal) and from late-born/secondary neuromasts (LII.1, D2), labeled by red-dextran (D-G) or by SILL1 DNA injection $(\boldsymbol{H}, \boldsymbol{I})$, at $6 \mathrm{dpf}$ in a Tg[hspGFFDMC130A; UAS:EGFP] transgenic animals. All pictures are snapshots of the three-dimensional reconstruction of the central projections and the distal tip of the Mauthner cell's lateral dendrite (green). J, Quantification of the contacts between lateralis afferent neurons and the lateral dendrite of the Mauthner cell, sorted by labeling method and neuromast identity: early-born/primary neuromasts (L1, D1, terminal) and late-born/secondary neuromasts (LII.1, LII.2, LII.3, D2). $\boldsymbol{K}$, Central projections from afferent neurons labeled by SILL1 DNA injection in Tg[hspGFFDMC130A; UAS:EGFP] transgenics, at 4, 7 and $10 \mathrm{dpf}$. Two lateralis afferent neurons are labeled; one contacts the Mauthner cell whereas the other does not. $L$, Central axons from afferent neurons projecting from terminal neuromasts and labeled by red-dextran at $\sim 20 \mathrm{dpf}$, in the $H G n 39 D$ transgenic line (colored in red). The lateralis column is shown in red. Medial and lateral focal planes are shown. The asterisk indicates dorsal projections with an indentation. The red arrowhead indicates the ventrolateral projections without the indentation. 

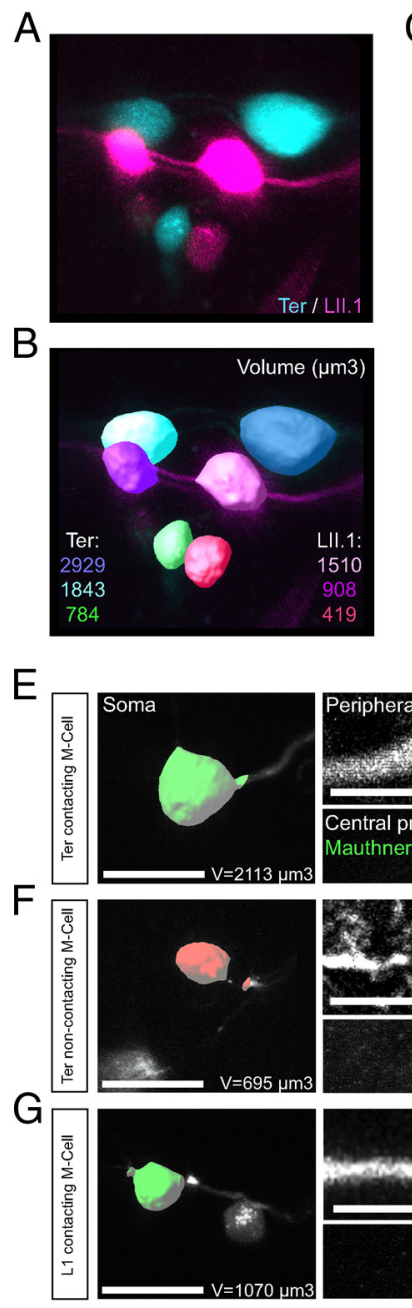

C

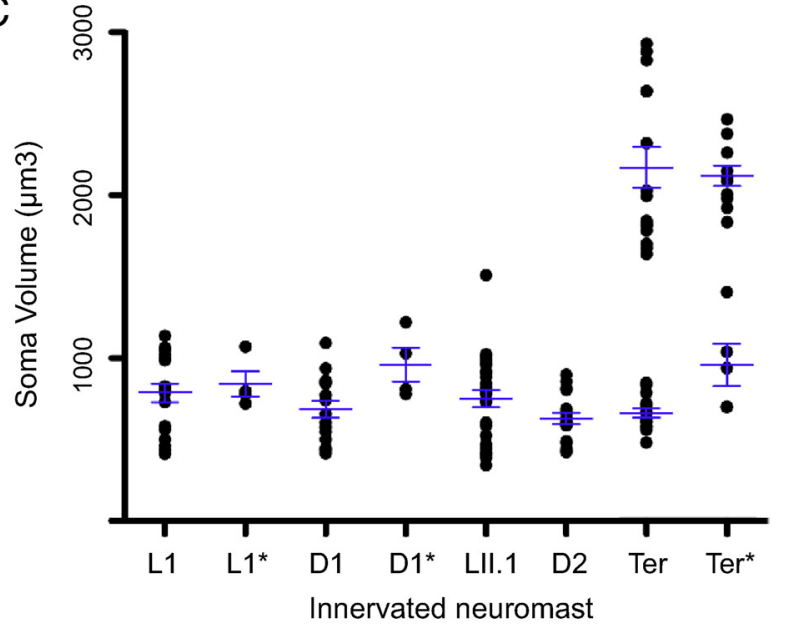

D

\begin{tabular}{|c|c|c|}
\hline \multirow{2}{*}{$\begin{array}{c}\text { Innervated } \\
\text { neuromast }\end{array}$} & \multicolumn{2}{|c|}{ Soma Volume $(\mu \mathrm{m} 3)$} \\
\hline & Dextran / & $\mathrm{DNA}^{*}$ \\
\hline \multirow[t]{2}{*}{ L 1} & $788 \pm 57$ & $842 \pm 78$ \\
\hline & $n=18$ & $n=4$ \\
\hline \multirow{2}{*}{ D 1} & $685 \pm 53$ & $960 \pm 103$ \\
\hline & $n=15$ & $n=4$ \\
\hline LII.1 & $\begin{array}{c}749 \pm 52 \\
n=27\end{array}$ & $\ldots$ \\
\hline \multirow[t]{2}{*}{ D2 } & $629 \pm 36$ & --. \\
\hline & & \\
\hline \multirow{2}{*}{$\begin{array}{c}\text { Ter } \\
\text { (large) }\end{array}$} & $2170 \pm 125$ & $2120 \pm 63$ \\
\hline & $n=15$ & $n=10$ \\
\hline \multirow{2}{*}{$\begin{array}{l}\text { Ter } \\
\text { (small) }\end{array}$} & $659 \pm 29$ & $953 \pm 130$ \\
\hline & $n=14$ & $n=5$ \\
\hline
\end{tabular}
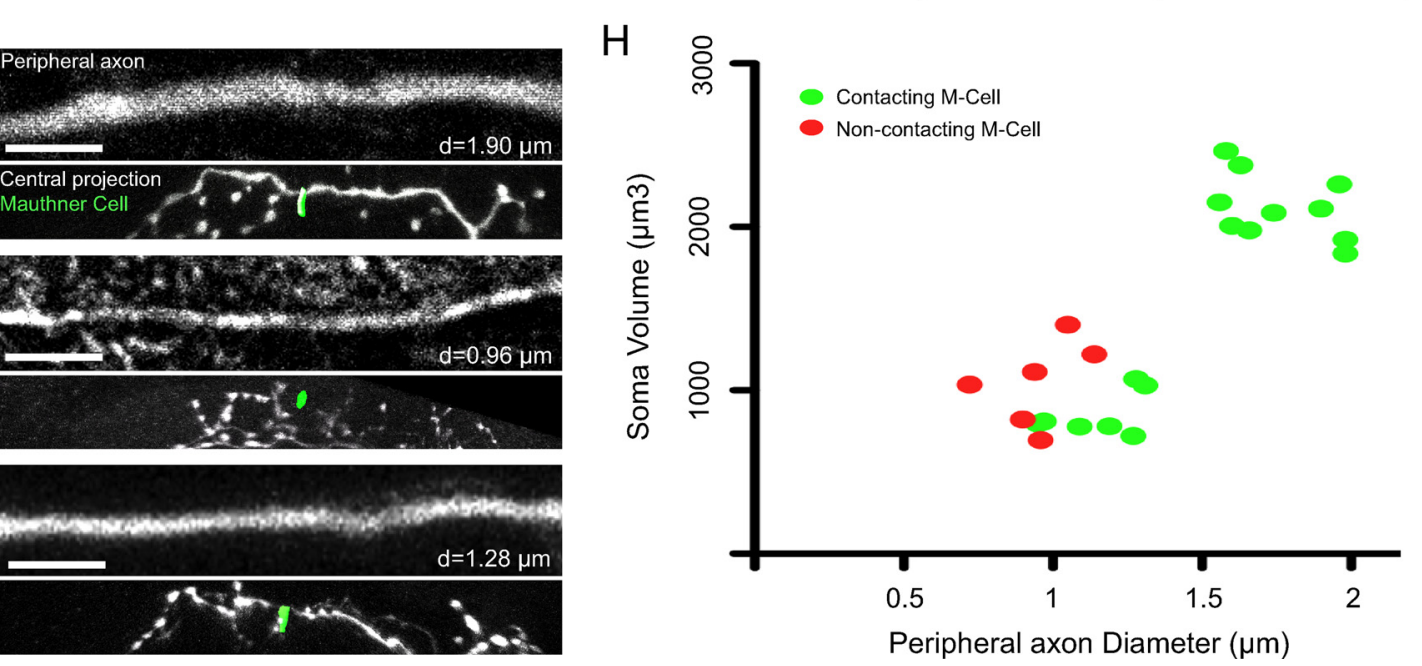

Figure 4. Neuronal subclassification based on soma volume and peripheral axon diameter. $A$, Posterior lateralis ganglion at $7 \mathrm{dpf}$ with somata from afferent neurons projecting from the terminal neuromasts labeled with red-dextran (false-colored blue) and neurons projecting from the Lll.1 neuromast labeled with magenta-dextran (magenta). $\boldsymbol{B}$, Surface reconstruction of each soma that was used for volumetric analysis. C, Quantification of soma volume $\left(\mu \mathrm{m}^{3}\right)$ at $7 \mathrm{dpf}$. Each dot represents the soma volume of an individual neuron. Data are grouped in the $x$-axis by both the innervated neuromast and the labeling method (no asterisk: dextran, asterisk: DNA). Averages and error bars (SEM) are shown in blue. Neurons projecting from the terminal neuromasts were split into two categories: large-soma $\left(>1500 \mu \mathrm{m}^{3}\right)$ and small-soma $\left(<1500 \mu \mathrm{m}^{3}\right)$ neurons. Averages and SEM were calculated separately for each category. $D$, Soma volume averages $\left(\mu \mathrm{m}^{3}\right.$, with SEM) at $7 \mathrm{dpf}$, grouped by both the innervated neuromast and the labeling method. E-G, Three examples of lateralis afferent neurons at $7 \mathrm{dpf}$, labeled by SILL1 DNA injection. Surface reconstructions for each soma are shown. Green and red indicates contacting and non-contacting the Mauthner cell, respectively. Peripheral axon images are focal planes. Snapshots of the three-dimensional reconstruction of the central projection and the distal tip of the Mauthner cell's lateral dendrite (green) are also shown. $\boldsymbol{H}$, Correlation between contacts with the Mauthner cell, soma volume ( $\mu \mathrm{m}^{3}$ ) and peripheral axon diameter $(\mu \mathrm{m})$ as an average of ten equidistant points along the axon at $7 \mathrm{dpf}$. Each dot represents the soma volume and peripheral axon diameter of an individual neuron, labeled by SILL1 DNA injection. In all the panels dorsal is up and anterior is left. Scale bars represent $5 \mu \mathrm{m}$ for panels showing peripheral axons, and $10 \mu \mathrm{m}$ for panels showing neuronal somata.

beled neurons by fluorescent dextrans at different stages of zebrafish development. This experiment confirmed that large neurons arrive to the target neuromast first (Fig. 5A, B). Lateralis neurogenesis in the zebrafish embryo occurs in two discrete waves, whose temporal sequence results in a strongly biased dorsoventral localization of neurons in the ganglion (Pujol-Martí et al., 2010; Sarrazín et al., 2010). To test the hypothesis that dorsal-most neurons belong to the first wave and have bigger somata, we developed BAIT, by which timed fluorescentprotein photoconversion dates cells, and dextran incorporation reveals their structure. BAIT showed that older neurons are larger and are mostly located in the dorsal aspect of the ganglion (Fig. $5 C, D)$. We have previously reported that the transgenic line hspGFF53A is one of the earliest markers lateralis afferent neurons, and that HGn39D begins to be expressed in the same neurons several hours later (Pujol-Martí et al., 2010). A reassessment of these transgenic lines showed that the fluorescent signal from HGn39D persists as neurons age, whereas that of hspGFF53A fades with time. Neurons located in the ventral aspect of the ganglion (late-born) expressed SILL1 (mCherry+) and hspGFF53A (EGFP+). However, hspGFF53A was downregulated in dorsally placed neurons (early-born), whereas SILL1 expression was maintained $(\mathrm{mCherry}(+) / \operatorname{EGFP}(-))$. The combination of this transgenics is therefore complementary to BAIT to date neurons in vivo. Using the triple transgenics $T g$ [SILL1; hspGFF53A; UAS:EGFP] injected with dextrans in terminal neuromasts we analyzed the soma and central projection of neurons of different ages by threedimensional confocal microscopy $(N=18)$ (Fig. $5 E-G)$. This experiment consistently revealed that the oldest neurons (mCherry $(+) /$ $\operatorname{EGFP}(-))$ were larger and localized to the ganglion's dorsum $(N=$ $11)$, whereas the youngest neurons $(\operatorname{mCherry}(+) / \operatorname{EGFP}(+))$ were smaller and localized to the ventral aspect of the ganglion $(N=7)$ (Fig. $5 E$ ). When we examined the central projections from the oldest neurons we observed that they were always characterized by and 
A

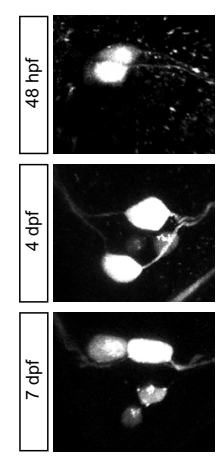

B

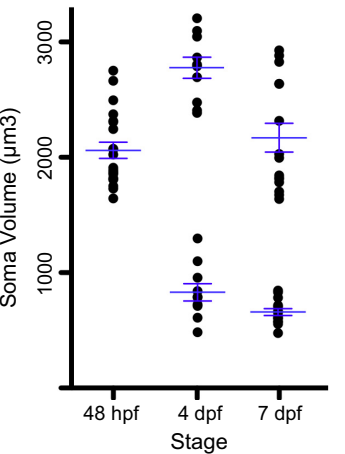

C

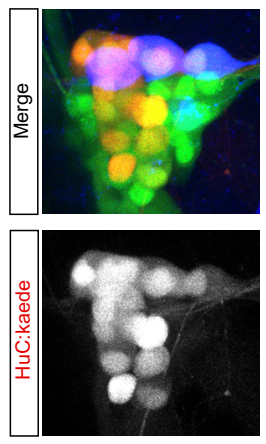

D

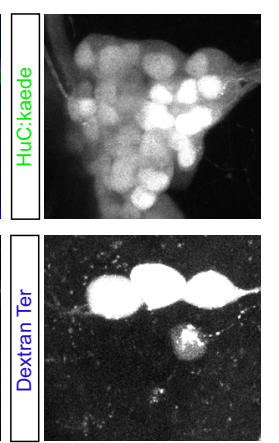

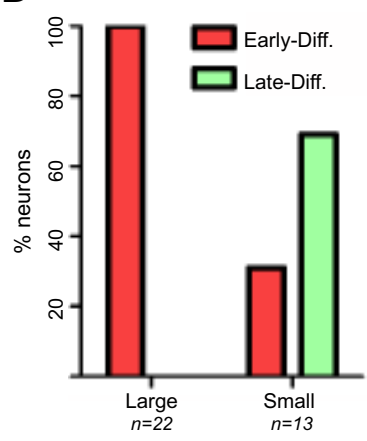

Soma Volume
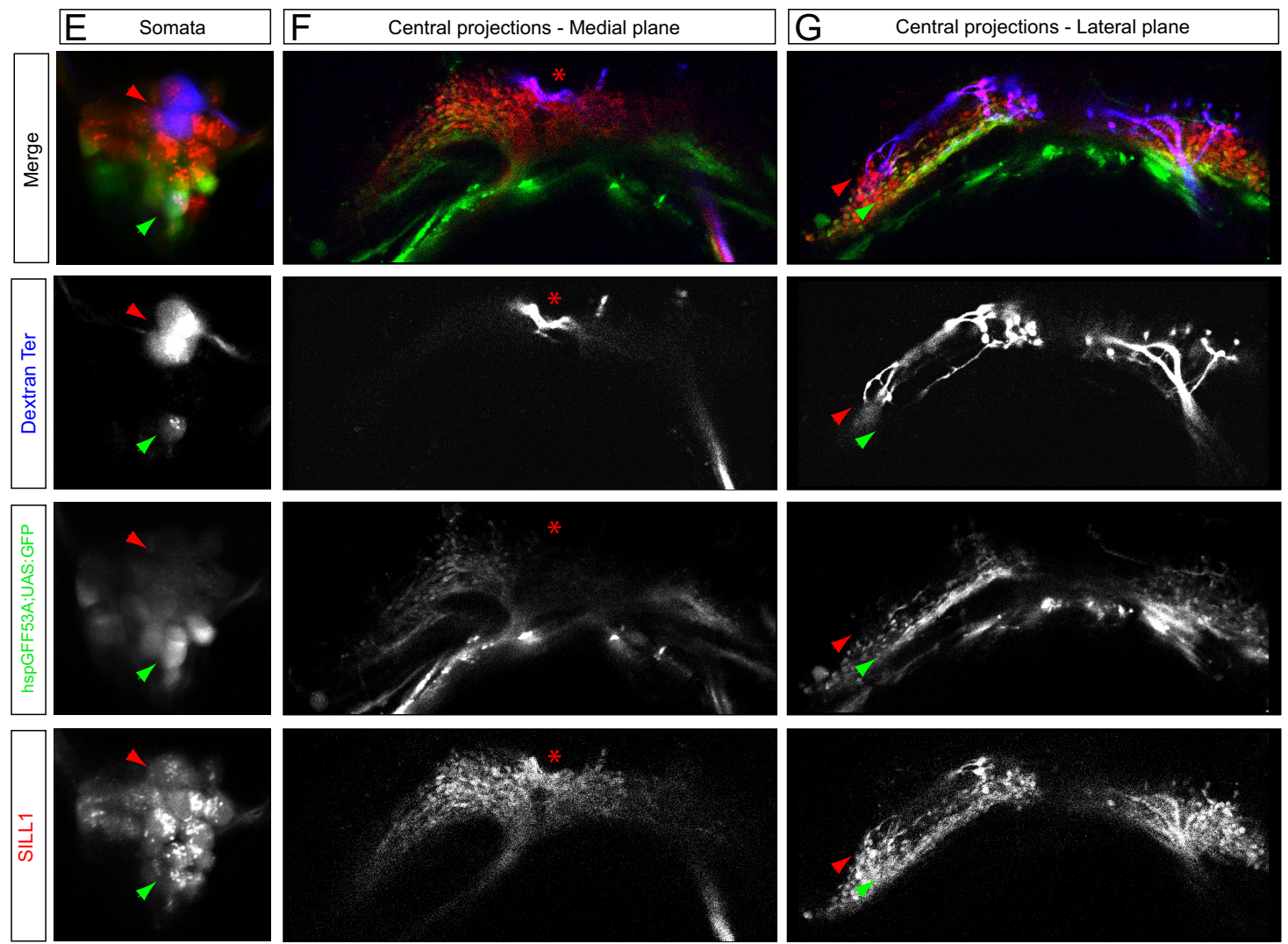

Figure 5. Simultaneous analysis of innervation/birthdate and soma volume of lateralis afferent neurons projecting from the terminal neuromasts. $A$, Posterior lateralis ganglion with somata from afferent neurons projecting from the terminal neuromasts labeled at different stages ( $48 \mathrm{hpf}, 4 \mathrm{dpf}$, and $7 \mathrm{dpf})$. $\boldsymbol{B}$, Quantification of soma volume ( $\mu \mathrm{m}^{3}$ ) from afferent neurons projecting from the terminal neuromasts labeled at different stages. Each dot represents the soma volume of an individual neuron. The data were grouped in the $x$-axis by the stage of labeling. Averages and error bars (SEM), shown in blue, were calculated separately for the large- and small-soma neurons categories. C, Maximal projection of a posterior lateralis ganglion at $5 \mathrm{dpf}$ resulting from a BAlT experiment. Kaede photoconversion was performed at $\sim 28 \mathrm{hpf}$. Early-born neurons (converted at $\sim 28 \mathrm{hpf}$ ) are green and red (yellow), whereas late-born neurons appear only green. Three large and one small soma from afferent neurons projecting from the terminal neuromasts were labeled by magenta-dextran uptake at $4 \mathrm{dpf}$. $\boldsymbol{D}$, Percentage of large and small somata from afferent neurons projecting from the terminal neuromasts of both types: early- and late-born. $\boldsymbol{E}-\boldsymbol{G}$, Confocal planes of somata $(\boldsymbol{E})$ and central projections $(\boldsymbol{F}, \boldsymbol{G})$ from lateralis afferent neurons projecting from the terminal neuromasts labeled by magenta-dextran at 6 dpf, in a Tg[SILL1; hspGFF53A; UAS:EGFP] triple transgenic. The red and green arrowheads indicate two large and one small soma, respectively. Central projections pictures: the asterisk indicates the indentation. Red and green arrowheads indicate dorsal and ventrolateral projections, respectively.

indentation in their rostral ramus and projected axons dorsally in the hindbrain, whereas youngest neurons always had ventrolateral projections (Fig. 5F,G). From these results we confirm that soma size and axonal projections correlate with the sequence of neuronal birth and differentiation (two-tailed Fisher's exact test, with a $p<0.05$ ).

The lateralis neural map develops in the absence of sensory input

We wanted to know whether evoked sensory activity played any role in neuronal subclassification or instructed their projection pattern in the hindbrain. For this purpose, we combined the injection of a magenta-dextran in terminal neuromasts whose large neurons always contact the Mauthner cell, and red dextran in a secondary neuromast that are devoid of neurons projecting to the Mauthner. These injections were made in wild-type animals and in mutants lacking hair-cell mechanoreception (homozygous mutant for tmie), or lacking hair cells in the neuromasts (homozygous mutant for atohla) (Fig. 6A-C) (Faucherre et al., 2010). We observed no differences in central projections or soma size between all three conditions, demon- 


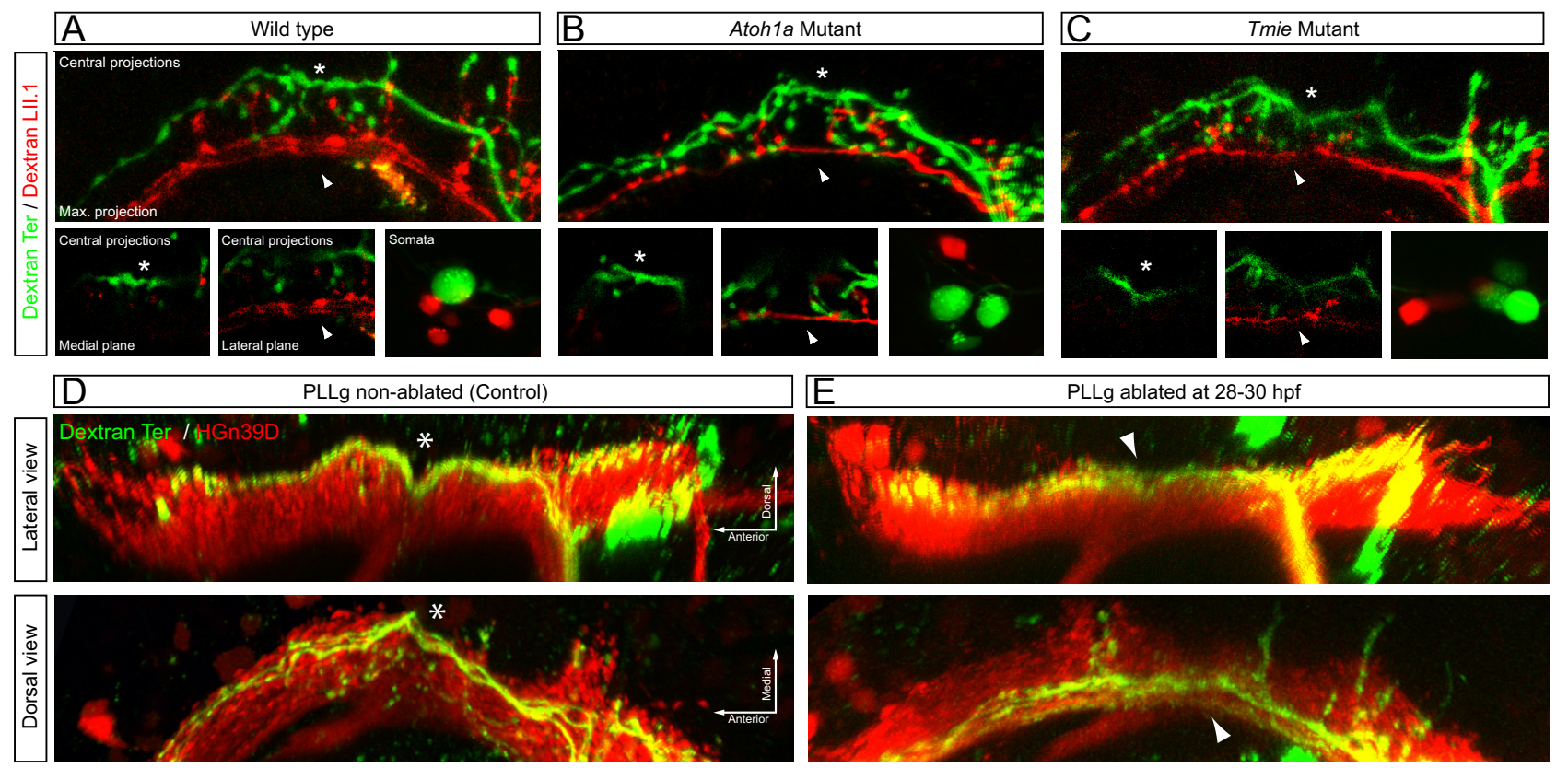

Figure 6. Analysis of the lateralis neural map in the absence of sensory input and in the absence of early-born neurons. $A-C$, Central projections and somata from lateralis afferent neurons projecting from the terminal neuromasts (in green, magenta-dextran) and the LII.1 neuromast (in red, red-dextran), in wild-type $(\boldsymbol{A})$ and homozygous mutants for atoh $1 a(\boldsymbol{B})$ and tmie $(\boldsymbol{C})$. Neurons were labeled at $6 \mathrm{dpf}$. Detailed views of both the indentation (asterisk) and the ventrolateral projections (arrowhead) are shown in medial and lateral focal planes, respectively. Top panels in $\boldsymbol{A}-\boldsymbol{C}$ are maximal projections. $\boldsymbol{D}, \boldsymbol{E}$, Central projections from afferent neurons projecting from the terminal neuromasts that were labeled by magenta-dextran in $H G n 39 D$ transgenics, whose posterior lateralis ganglion (PLLg) was laser ablated or non-ablated (control) at $28-30 \mathrm{hpf}$. Neurons were labeled at $6 \mathrm{dpf}$ by dextran uptake. Both lateral and dorsal views of the three-dimensional reconstruction of the central projections are shown. The lateralis column is shown in red. The asterisk indicates the indentation in the dorsal projections. The arrowhead indicates lateral projections without the indentation.

strating that evoked sensory input does not instruct neuronal subclassification or the pattern of central projections. Another mechanism that could control map topology is competition, whereby axons from early-born neurons repel those from neurons born later from contacting the Mauthner cell. To test this hypothesis it is useful to observe the axonal projections from late-born neurons in the absence of early-born. If competition between neuronal subclasses does not play a role in axonal projections, late-born neurons from experimental animals should be indistinguishable from those under normal conditions. If, alternatively, interactions between axons are responsible for the differences, the projections of late-born neurons should be altered when early-born are absent. We tested this possibility by eliminating early-born neurons in HGn39D transgenics by laser ablation at 28-30 hpf, a stage in development between the differentiation of both neuronal subclasses. Subsequently, magenta-dextran was injected in terminal neuromasts and central axonal projections were analyzed by three-dimensional confocal microscopy. In all cases analyzed the ablation of early-born neurons did not modify the axonal projections from late-born neurons (Fig. 6D,E). Collectively, these results demonstrate that neither axonal competition, nor evoked sensory activity play a significant role during the assembly of the lateralis neural map.

\section{Discussion}

The piscine lateralis afferent neurons assemble a somatotopic neural map, in which axons are reproducibly positioned along a dorsoventral axis in the hindbrain (Claas and Münz, 1981; Alexandre and Ghysen, 1999; Ghysen and Dambly-Chaudière, 2007). Although somatotopy probably forms a neuroanatomical code of the external hydrodynamic field, it is not well understood how animals use it to command behaviors. In this study we used neuronal labeling and image registration to reveal two classes of lateralis afferent neurons defined by birth order, soma size and location, central projections and contacts with an identified output neuron, to define a dimorphic sensorineural map. Next, we discuss our results and make predictions on how lateralis map topology may underlie appropriate behavioral responses according to the biological relevance of mechanosensory input.

Lateralis afferent neurons are extremely interesting because they can shed light on the mechanisms that initiate different behavioral programs by the same sensory modality. Many studies have firmly demonstrated a role of the lateral line in navigation, schooling and prey detection (Dijkgraaf, 1963; Montgomery et al., 1997, 2000; Coombs et al., 1998; Bleckmann, 2008; Coombs and Patton, 2009). It has also been proposed that the lateral line can send powerful inputs to the Mauthner cell to trigger the C-start escape response (McHenry et al., 2009). The optical transparency, external and fast development of the zebrafish, coupled with the accessibility and anatomical simplicity of its lateral line provides a powerful model to study the neuroanatomical bases of these contrasting behaviors. Because an essential part of investigations toward this aim is the careful characterization of neuronal connectivity, we generated two transgenic zebrafish lines for targeted gene expression. The hspGFFDMC130A line contains the Gal4 transcriptional activator stably integrated in the genome (Nagayoshi et al., 2008). We demonstrate that hspGFFDMC130A can express fluorescent markers in the Mauthner cell and few other tissues. We also generated the SILL1 transgenics that expresses the mCherry red-fluorescent protein in all the lateralis afferent neurons. Single-neuron labeling with the SILL1 construct revealed unexpected neuronal diversification and neuralmap dimorphism. In a divergent neural submap, each neuron forms $\sim 60$ boutons that concentrate a well characterized presyn- 
aptic marker. If we assume that each bouton represents a synaptic contact with one output neuron, the posterior lateral line has no more than 2,400 second-order targets in the zebrafish larva (Fame et al., 2006; Sassa et al., 2007). In a convergent submap, many lateralis neurons directly contact the lateral dendrite of the Mauthner cell.

What determines map dimorphism and target selectivity? One possibility is that sensory activity plays an instructive role (Luo and Flanagan, 2007). However, our results from the analysis of two types of mutants-a strong loss-of-function in Tmie that blocks hair-cell mechanoreception, and a loss-of-function in Atoh1a that prevents the development of hair cells in neuromasts-indicate that sensory activity is not a major force in sculpting the lateralis neural map. Axonal competition has been shown to influence neural mapping in several systems. However, our selective ablation of one neuronal subpopulation did not alter the projection of the remaining neurons. Therefore, inter-class axonal competition does not appear to instruct map topology.

We combined our transgenic lines with dextran injections for anatomical tracing to develop BAIT. One caveat of BAIT is that it does not reveal the actual birth dates of cells, but their relative age. Therefore, we made the assumption that neurons differentiate at similar rates once they have fate-committed. In doing so, we demonstrate a correlation between the sequence of afferent neurogenesis and central projections, and that only early-born neurons converge on the Mauthner cell. Although we cannot currently rule out that molecular gradients in the target area refine map topology much the same way as they do in other sensory systems, our findings support a strong contribution of neurogenic timing in lateralis neural map dimorphism and projection pattern (Fariñas et al., 2001; Luo and Flanagan, 2007; Clandinin and Feldheim, 2009; Feldheim and O'Leary, 2010; Schuster et al., 2010). Such strategy is not likely to be unique to the lateral line, however. For example, mechanosensory neurons of the wing or photoreceptor neuron of the eye in Drosophila also produce tiered central projections based on their time of development (Palka et al., 1986; Morey et al., 2008; Petrovic and Hummel, 2008). Earlier studies in Xenopus tested the involvement of timing in generating the topographic organization of the neuronal projection from the retina to the tectum, and concluded that it likely plays a permissive rather than an instructive role in axonal organization (Holt, 1984). It would be interesting to test permissive versus instructive roles of timing in the lateral line.

Neuromasts are directionally sensitive by virtue of the planar polarization of their constituent hair cells. Posterior neuromasts have orthogonal planar orientations. Parallel neuromasts develop earlier from a first primordium and are called "primary neuromasts." Perpendicular neuromasts develop subsequently from independent primordia and are called "secondary" (López-Schier et al., 2004; Ghysen and DamblyChaudière, 2007; Sarrazín et al., 2010). Our study demonstrates that waves of afferent neurogenesis accompany the protracted development of the neuromasts. Primary neuromasts project early-born neurons that diverge along the hindbrain but some of which also converge on the Mauthner cell. Secondary neuromasts, by contrast, only project late-born neurons that do not converge on the Mauthner. A potentially equivalent relationship between developmental timing and neuronal projection patterns has been reported for the segregation of afferent projections for the otic, lateralis and ampullary organs in the axolotl (Fritzsch et al., 2005). In this case, however, the relationship is between organs rather than within each organ.

Afferent input to the Mauthner from primary neuromasts may be an elegant strategy to couple development with behavior because the earliest born neurons and neuromasts could allow the animal to use the lateral line for escape responses before it is able to swim. We show that in the zebrafish larva, terminal neuromasts are innervated by large-diameter afferent axons. The conduction velocity of myelinated axons in vertebrates increases linearly with their diameter (Hursh, 1939; Holmes, 1941; Goldman and Albus, 1968). Therefore, early-born neurons projecting from terminal neuromasts are likely to be fast conducing, making them well suited to produce the first and fastest lateral-line stimulus for the C-start response. This also suggests that terminal neuromasts have a disproportionate relevance in the escape behavior mediated by the Mauthner cell. Interestingly, the trout also shows regional segregation of lateralis neurons relative to axon diameter and conduction velocity, which can compensate for the increased axonal length in large animals (Schellart and Kroese, 2002).

Are terminal neuromasts enough to trigger the C-start response? Unlike neurons from other parts of the lateral line, each large neuron innervates up to three terminal neuromasts, which may increase their depolarization probability. This presents clear survival advantages because terminal neuromasts may suffice to trigger a C-start reaction by sending strong depolarizing inputs to the Mauthner cell with very short latencies. However, in general fish should not startle by nonthreatening stimuli, which predicts that early-born neurons should have lower sensitivity than late-born. Behaviors such as navigation, rheotaxia and schooling necessitate continuous input and probably have lower activating thresholds that the C-start. Afferent neurons with different excitability and conduction velocities have been reported for the posterior lateral line of the goldfish (Fukuda, 1974). Also, in cichlids, lateralis afferent neurons with higher rate of spontaneous discharge are more sensitive (Münz, 1985). Interestingly, the rate of spontaneous activity of individual afferent neurons in the zebrafish larva varies along the lateral line (Liao, 2010). If these observations are extrapolated and combined with our findings, one emergent possibility is that the C-start will only be triggered by the coincident input on the Mauthner cell from "high sensitivity/low conduction velocity" and "lower sensitivity/high conduction velocity" neuronal classes. This model will safeguard the animal from startling upon stimuli that would depolarize one neuronal subclass but not the other, and is reminiscent of the escape strategy of crayfish, in which a mechanosensory stimulus activates parallel neuronal pathways with different reaction times that trigger the startle when arriving coincidently to an output command neuron (Reichert and Wine, 1982; Mellon and Christison-Lagay, 2008). However, more complex processing of hydrodynamic stimuli is possible, with multiple neuromasts contributing to the probability of depolarizing excitatory signals from the lateral line to the Mauthner cell (Korn et al., 1974).

Although spatiotemporal resolution may involve secondary neuromasts, they may not contribute to the C-start response because of their discrete location and their insensitivity to the direction of propagation of most of the water flow generated by submerged predators. This may explain why their afferent neurons do not form monosynaptic contacts with the Mauthner cell. This begs the question of whether somatotopy has any functional relevance to the C-start reaction. Currently, we favor the hypoth- 
esis that lateralis somatotopy does not play a major role in the escape behavior of the zebrafish larva. Thus, the lateral line would sacrifice spatial accuracy for sensitivity and response speed. For navigation, however, somatotopy is likely to be essential. Thus, the lateral line may assemble a convergent submap for speed and a divergent submap for accuracy. The combined activity of neurons can code for the entire range of stimuli (spectrum) that a sensory organ can acquire. Variation in the sensitivity of individual sensory receptors within the organ can subtract aspects of a complex stimulus to direct behavior. This subdivision of work is called range fractionation (Cohen, 1963). However, variations in sensory transducers can also impact information processing. Therefore, the next challenge is to determine whether sensorineural map dimorphism in the lateral line form the bases of range fractionation by this sensory system (Voigt et al., 2000; Braun et al., 2002).

\section{References}

Alexandre D, Ghysen A (1999) Somatotopy of the lateral line projection in larval zebrafish. Proc Natl Acad Sci U S A 96:7558-7562.

Asakawa K, Suster ML, Mizusawa K, Nagayoshi S, Kotani T, Urasaki A, Kishimoto Y, Hibi M, Kawakami K (2008) Genetic dissection of neural circuits by Tol 2 transposon-mediated Gal4 gene and enhancer trapping in zebrafish. Proc Natl Acad Sci U S A 105:1255-1260.

Bleckmann H (2008) Peripheral and central processing of lateral line information. J Comp Physiol A Neuroethol Sens Neural Behav Physiol 194:145-158.

Bleckmann H, Zelick R (2009) Lateral line system of fish. Integr Zool $4: 13-25$.

Braun CB, Coombs S, Fay RR (2002) What is the nature of multisensory interaction between octavolateralis sub-systems? Brain Behav Evol 59:162-176.

Claas B, Münz H (1981) Projection of lateral line afferents in a teleost's brain. Neurosci Lett 23:287-290.

Clandinin TR, Feldheim DA (2009) Making a visual map: mechanisms and molecules. Curr Opin Neurobiol 19:174-180.

Cohen MJ (1963) The crustacean myochordotonal organ as a proprioceptive system. Comp Biochem Physiol 16:223-243.

Coombs S, Patton P (2009) Lateral line stimulation patterns and prey orienting behavior in the Lake Michigan mottled sculpin (Cottus bairdi). J Comp Physiol A Neuroethol Sens Neural Behav Physiol 195:279-297.

Coombs S, Mogdans J, Halstead M, Montgomery J (1998) Transformation of peripheral inputs by the first-order lateral line brainstem nucleus. J Comp Physiol A 182:606-626.

Dijkgraaf S (1963) The functioning and significance of the lateral line organs. Biol Rev 38:51-105.

Eaton RC, Farley RD, Kimmel CB, Schabtach E (1977) Functional development in the Mauthner cell system of embryos and larvae of the zebra fish. J Neurobiol 8:151-172.

Fame RM, Brajon C, Ghysen A (2006) Second-order projection from the posterior lateral line in the early zebrafish brain. Neural Dev 1:4.

Fariñas I, Jones KR, Tessarollo L, Vigers AJ, Huang E, Kirstein M, de Caprona DC, Coppola V, Backus C, Reichardt LF, Fritzsch B (2001) Spatial shaping of cochlear innervation by temporally regulated neurotrophin expression. J Neurosci 21:6170-6180.

Faucherre A, Pujol-Martí J, Kawakami K, López-Schier H (2009) Afferent neurons of the zebrafish lateral line are strict selectors of hair-cell orientation. PLoS One 4:e4477.

Faucherre A, Baudoin JP, Pujol-Martí J, López-Schier H (2010) Multispectral four-dimensional imaging reveals that evoked activity modulates peripheral arborization and the selection of plane-polarized targets by sensory neurons. Development 137:1635-1643.

Feldheim DA, O'Leary DD (2010) Visual map development: bidirectional signaling, bifunctional guidance molecules, and competition. Cold Spring Harb Perspect Biol 2:a001768.

Fritzsch B, Gregory D, Rosa-Molinar E (2005) The development of the hindbrain afferent projections in the axolotl: evidence for timing as a specific mechanism of afferent fiber sorting. Zoology (Jena) 108:297-306
Fukuda J (1974) Fiber composition of the posterior lateral-line nerve of goldfish, investigated by electrophysiological and microscopical techniques. J Comp Neurol 155:203-217.

Ghysen A, Dambly-Chaudière C (2007) The lateral line microcosmos. Genes Dev 21:2118-2130.

Gleason MR, Nagiel A, Jamet S, Vologodskaia M, López-Schier H, Hudspeth AJ (2009) The transmembrane inner ear (Tmie) protein is essential for normal hearing and balance in the zebrafish. Proc Natl Acad Sci U S A 106:21347-21352.

Goldman L, Albus JS (1968) Computation of impulse conduction in myelinated fibers; theoretical basis of the velocity-diameter relation. Biophys J 8:596-607.

Holmes W (1941) The structure and conduction velocity of the medullated nerve fibres of prawns. J Exp Biol 18:50-54.

Holt CE (1984) Does timing of axon outgrowth influence initial retinotectal topography in Xenopus? J Neurosci 4:1130-1152.

Hua JY, Smear MC, Baier H, Smith SJ (2005) Regulation of axon growth in vivo by activity-based competition. Nature 434:1022-1026.

Hursh JB (1939) Conduction velocity and diameter of nerve fibers. Am J Physiol 127:131-139.

Kimmel CB, Sessions SK, Kimmel RJ (1981) Morphogenesis and synaptogenesis of the zebrafish Mauthner neuron. J Comp Neurol 198: $101-120$.

Kimmel CB, Hatta K, Metcalfe WK (1990) Early axonal contacts during development of an identified dendrite in the brain of the zebrafish. Neuron 4:535-545.

Korn H, Faber DS, Mariani J (1974) [Existence of projections of posterior nerves from the lateral line on the Mauthner cell; their antagonistic effect on the activation of this neuron by vestibular afferences]. C R Acad Sci Hebd Seances Acad Sci D 279:413-416.

Kwan KM, Fujimoto E, Grabher C, Mangum BD, Hardy ME, Campbell DS, Parant JM, Yost HJ, Kanki JP, Chien CB (2007) The Tol2kit: a multisite gateway-based construction kit for Tol2 transposon transgenesis constructs. Dev Dyn 236:3088-3099.

Liao JC (2010) Organization and physiology of posterior lateral line afferent neurons in larval zebrafish. Biol Lett 6:402-405.

López-Schier H, Starr CJ, Kappler JA, Kollmar R, Hudspeth AJ (2004) Directional cell migration establishes the axes of planar polarity in the posterior lateral-line organ of the zebrafish. Dev Cell 7:401-412.

Luo L, Flanagan JG (2007) Development of continuous and discrete neural maps. Neuron 56:284-300.

McHenry MJ, Feitl KE, Strother JA, Van Trump WJ (2009) Larval zebrafish rapidly sense the water flow of a predator's strike. Biol Lett 5:477-479.

Mellon D Jr, Christison-Lagay K (2008) A mechanism for neuronal coincidence revealed in the crayfish antennule. Proc Natl Acad Sci U S A 105:14626-14631.

Montgomery J, Baker C, Carton G (1997) The lateral line can mediate rheotaxis in fish. Nature 389:960-963.

Montgomery J, Carton G, Voigt R, Baker C, Diebel C (2000) Sensory processing of water currents by fishes. Philos Trans R Soc Lond B Biol Sci 355:1325-1327.

Morey M, Yee SK, Herman T, Nern A, Blanco E, Zipursky SL (2008) Coordinate control of synaptic-layer specificity and rhodopsins in photoreceptor neurons. Nature 456:795-799.

Münz H (1985) Single unit activity in the peripheral lateral-line system of the cichlid fish Sarotherodon niloticus. J Comp Physiol A Neuroethol Sens Neural Behav Physiol 157:555-568.

Nagayoshi S, Hayashi E, Abe G, Osato N, Asakawa K, Urasaki A, Horikawa K, Ikeo K, Takeda H, Kawakami K (2008) Insertional mutagenesis by the Tol2 transposon-mediated enhancer trap approach generated mutations in two developmental genes: tcf7 and synembryn-like. Development 135:159-169.

Palka J, Malone MA, Ellison RL, Wigston DJ (1986) Central projections of identified Drosophila sensory neurons in relation to their time of development. J Neurosci 6:1822-1830.

Peñagarikano O, Abrahams BS, Herman EI, Winden KD, Gdalyahu A, Dong H, Sonnenblick LI, Gruver R, Almajano J, Bragin A, Golshani P, Trachtenberg JT, Peles E, Geschwind DH (2011) Absence of CNTNAP2 leads to epilepsy, neuronal migration abnormalities, and core autismrelated deficits. Cell 147:235-246.

Petrovic M, Hummel T (2008) Temporal identity in axonal target layer recognition. Nature 456:800-803. 
Pujol-Martí J, Baudoin JP, Faucherre A, Kawakami K, López-Schier H (2010) Progressive neurogenesis defines lateralis somatotopy. Dev Dyn 239:1919-1930

Reichert H, Wine JJ (1982) Neural mechanisms for serial order in a stereotyped behaviour sequence. Nature 296:86-87.

Sarrazín AF, Nuñez VA, Sapède D, Tassin V, Dambly-Chaudière C, Ghysen A (2010) Origin and early development of the posterior lateral line system of zebrafish. J Neurosci 30:8234-8244.

Sassa T, Aizawa H, Okamoto H (2007) Visualization of two distinct classes of neurons by gad2 and zicl promoter/enhancer elements in the dorsal hindbrain of developing zebrafish reveals neuronal connectivity related to the auditory and lateral line systems. Dev Dyn 236:706-718.

Sato A, Koshida S, Takeda H (2010) Single-cell analysis of somatotopic map formation in the zebrafish lateral line system. Dev Dyn 239:2058-2065.

Sato T, Takahoko M, Okamoto H (2006) HuC:Kaede, a useful tool to label neural morphologies in networks in vivo. Genesis 44:136-142.
Schellart NA, Kroese AB (2002) Conduction velocity compensation for afferent fiber length in the trunk lateral line of the trout. J Comp Physiol A Neuroethol Sens Neural Behav Physiol 188:561-576.

Schuster K, Dambly-Chaudière C, Ghysen A (2010) Glial cell line-derived neurotrophic factor defines the path of developing and regenerating axons in the lateral line system of zebrafish. Proc Natl Acad Sci U S A 107:19531-19536.

Sheskin DJ (2004) Handbook of parametric and nonparametric statistical procedures, Ed 3. Boca Raton, FL: Chapman and Hall/CRC.

Voigt R, Carton AG, Montgomery JC (2000) Responses of anterior lateral line afferent neurones to water flow. J Exp Biol 203:24952502.

Zottoli SJ, Van Horne C (1983) Posterior lateral line afferent and efferent pathways within the central nervous system of the goldfish with special reference to the Mauthner cell. J Comp Neurol 219:100 111 